\title{
Fuzzy Nonlinear Programming with Applications in Decision Making
}

\author{
Tin-Chih Toly Chen, ${ }^{1}$ Deng-Feng Li, ${ }^{2}$ T. Warren Liao, ${ }^{3}$ and Yi-Chi Wang ${ }^{1}$ \\ ${ }^{1}$ Department of Industrial Engineering and Systems Management, Feng Chia University, Taichung 407, Taiwan \\ ${ }^{2}$ School of Management, Fuzhou University, Fujian 350108, China \\ ${ }^{3}$ Department of Mechanical and Industrial Engineering, Louisiana State University, Baton Rouge, LA 70803, USA
}

Correspondence should be addressed to Tin-Chih Toly Chen; tcchen@fcu.edu.tw

Received 14 November 2013; Accepted 14 November 2013; Published 23 January 2014

Copyright (C) 2014 Tin-Chih Toly Chen et al. This is an open access article distributed under the Creative Commons Attribution License, which permits unrestricted use, distribution, and reproduction in any medium, provided the original work is properly cited.

Fuzzy set theory provides an intuitive and computationally simple way to deal with uncertain and ambiguous properties. Decisions represented in terms of fuzzy sets also offer flexibility in their implementations. On the other hand, nonlinear programming relaxes the strict assumptions and constraints in linear programming and hence is more practicable to handle many decision making problems, which are better represented in the form of nonlinear programming models. Combining fuzzy set theory with nonlinear programming enables it to address the issue of uncertain parameters; the resulting fuzzy nonlinear programming problem involving fuzzy parameters can be viewed as an even more practicable approach than the conventional nonfuzzy one. In light of advanced computing systems, fuzzy nonlinear programming becomes one of the most promising approaches to solve practical application problems. The purpose of this special issue is to provide recent advances in developing fuzzy nonlinear programming methods and their applications to practicable and flexible decision making. The target audiences are researchers in fuzzy mathematics, operations research, information management, and system engineering, as well as practicing managers/engineers. After a strict review process, ten articles from researchers around the world were finally accepted. A brief summary of each is described below.

J. d. D. M. Silva et al. discussed the properties of pfuzzy dynamical systems that are variational systems of which dynamic behaviors are regulated by a Mamdani-type fuzzy system. They used a case study to illustrate a 1-dimensional p-fuzzy dynamical system and presented some theorems on the conditions of existence and uniqueness of stationary points.

H.-R. Tsai and T. Chen proposed a fuzzy nonlinear programming (FNLP) approach for optimizing the scheduling performance of a four-factor fluctuation smoothing rule in a wafer fabrication factory. The proposed methodology considered the uncertainty in the remaining cycle time of a job and optimized a fuzzy four-factor fluctuation-smoothing rule to sequence the jobs in front of each machine. The fuzzy four-factor fluctuation-smoothing rule had five adjustable parameters, the optimization of which resulted in a FNLP problem.

Most preferred ordered weighted average (MP-OWA) operators are a new kind of neat OWA operators in the aggregation operator families. It considers the preferences of all alternatives across the criteria and provides unique aggregation characteristics in decision making. X. Sang and X. Liu established the parametric form of the MP-OWA operator to deal with uncertain preference information, including the most commonly used maximum, minimum, and average aggregation operators. A special form of the parametric MPOWA operator with the power function was also proposed in their study.

The Bonferroni mean (BM) operator is an important aggregation technique which reflects the correlation of aggregated arguments. J. H. Park and E. J. Park studied the desirable properties of the fuzzy Bonferroni harmonic mean (FBHM) operator and the fuzzy ordered Bonferroni harmonic mean (FOBHM) operator. To consider the correlation of any three 
aggregated arguments, J. H. Park and E. J. Park developed the generalized fuzzy weighted Bonferroni harmonic mean (GFWBHM) operator and the generalized fuzzy ordered weighted Bonferroni harmonic mean (GFOWBHM) operator. Based on these new operators, they proposed a new approach to multiple-attribute group decision making and illustrated the new approach with a practical example.

J. Gang et al. studied a multiproject resource allocation problem in a bilevel organization. To solve the problem, a bilevel multiproject resource allocation model with fuzzy or random variables was established, in which the opinions of decision makers from two levels were considered. On the upper level, the company manager aims to allocate the company's resources to multiple projects to minimize the total costs. On the lower level, each project manager attempts to schedule their resource-constrained projects, with minimization of the project duration as the main objective. To search for the optimal solution, a hybrid approach of adaptive particle swarm optimization, adaptive hybrid genetic algorithm, and fuzzy random simulation was proposed.

Y.-C. Lin and T. Chen created an intelligent locationaware service, in which a timely service was recommended to the user without changing the user's pace. To the user, there were two goals to achieve: one is to reach the service location just in time and the other is to get to the destination as soon as possible. To optimize the two objectives at the same time and to consider the uncertainty in the dynamic environment, a biobjective fuzzy integer-nonlinear programming problem was formulated and solved.

L. Wang et al. defined the correlation measures of dual hesitant fuzzy sets (DHFSs) and discussed their properties. A direct transfer algorithm for complex matrix synthesis was also proposed that helps to construct an equivalent correlation matrix for clustering DHFSs. L. Wang et al. also proved that the direct transfer algorithm is equivalent to the transfer closure algorithm, but its asymptotic time complexity and space complexity are superior to the latter.

J. Zhang extended the concepts of preinvex and invex to the interval-valued functions. Under the assumption of invexity, the Karush-Kuhn-Tucker optimality of sufficient and necessary conditions was also proved for interval-valued nonlinear programming problems. J. Zhang also proved the Wolfe duality theorem for invex interval-valued nonlinear programming problems, based on the concept of having no duality gaps in weak and strong senses.

Fuzzy measures and fuzzy integrals have been successfully used in many real applications. However, the determination of fuzzy measures is still a challenging task. L. Chen, Z.-T. Gong, and G. Duan proposed a more generalized type of fuzzy measures by means of genetic algorithm and the Choquet integral. In this paper, they firstly defined the $\sigma-\lambda$ rule then defined and characterized the Choquet integrals of interval-valued functions and fuzzy functions based on the $\sigma-\lambda$ rule.

X. Zhou and Q. Li defined an accuracy function of hesitant fuzzy elements (HFEs) and developed a new method to compare two HFEs. Then, based on Einstein operators, they gave some new operational laws on HFEs and some desirable properties of these operations. Several new hesitant fuzzy aggregation operators, including the hesitant fuzzy Einstein weighted geometric $\left(\mathrm{HFEWG}_{\varepsilon}\right.$ ) operator and the hesitant fuzzy Einstein ordered weighted geometric $\left(\mathrm{HFEOWG}_{\varepsilon}\right)$ operator, were also developed as the extensions of the weighted geometric operator and the ordered weighted geometric (OWG) operator with hesitant fuzzy information, respectively.

\section{Acknowledgment}

We sincerely thank all the authors and reviewers for their valuable contributions to this special issue.

Tin-Chih Toly Chen Deng-Feng Li

T. Warren Liao

Yi-Chi Wang 


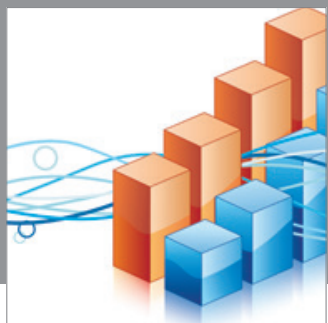

Advances in

Operations Research

mansans

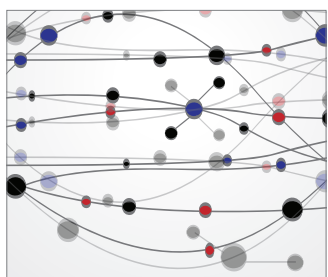

The Scientific World Journal
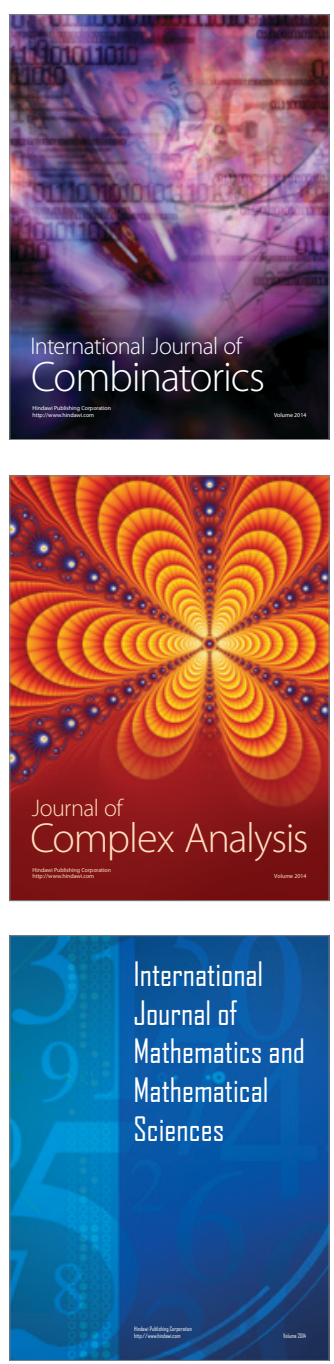
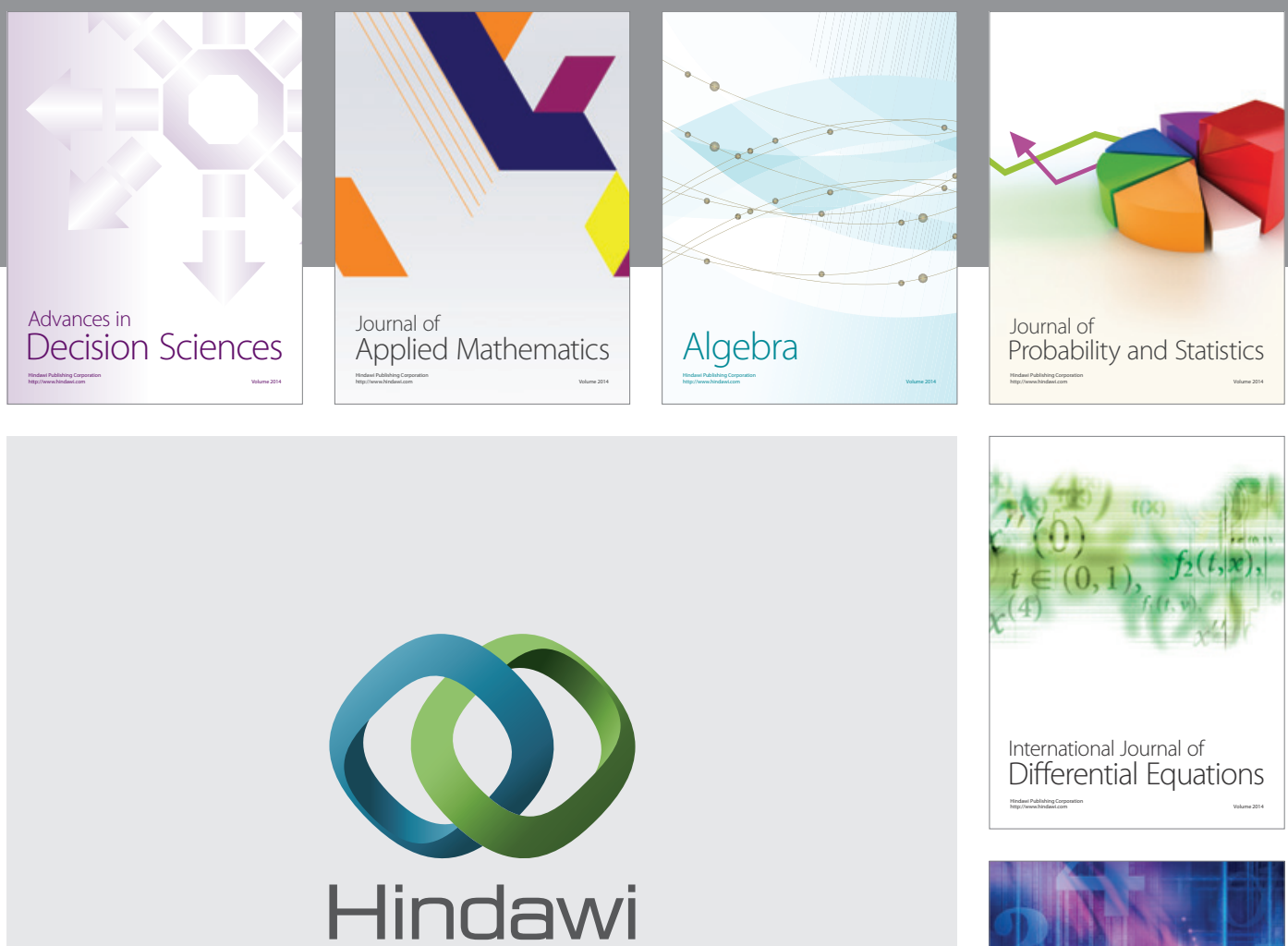

Submit your manuscripts at http://www.hindawi.com
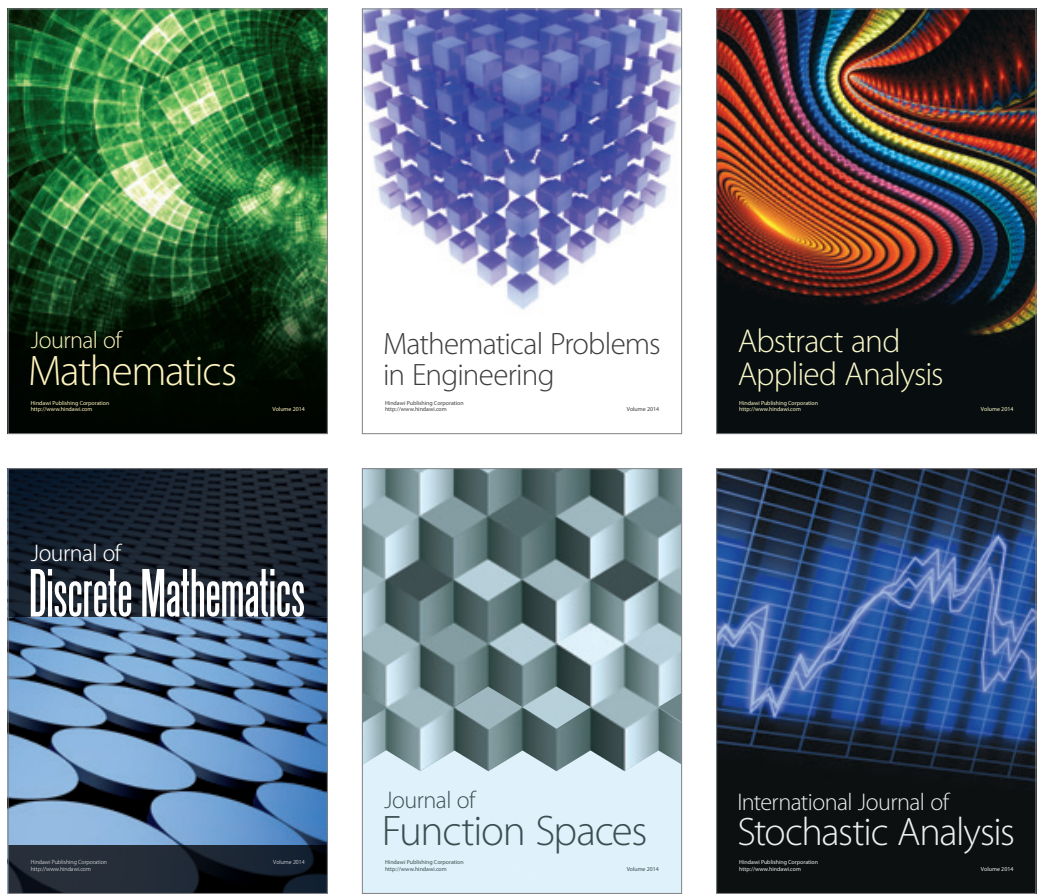

Journal of

Function Spaces

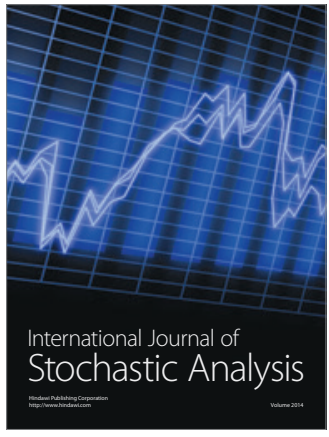

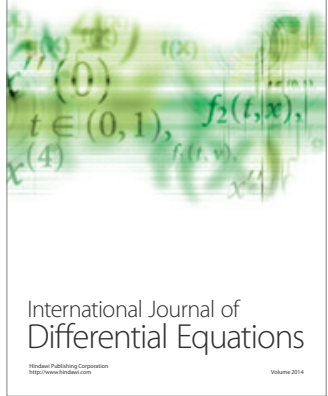
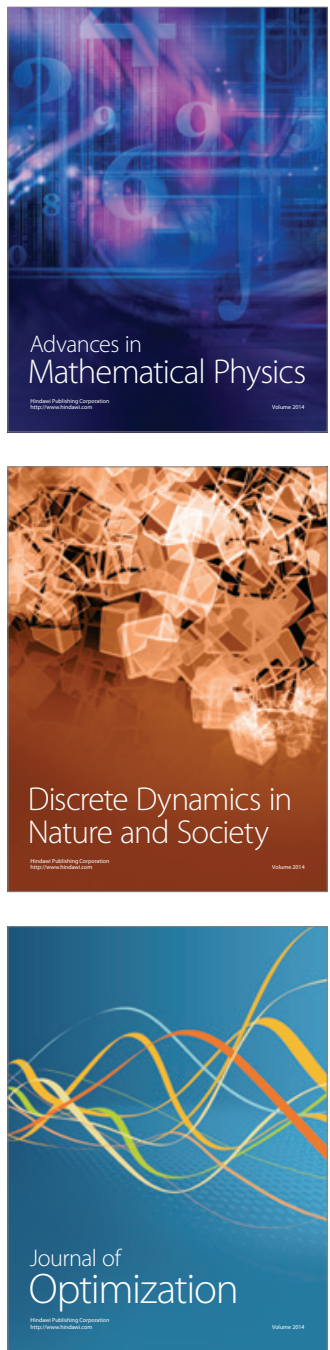